\title{
Preliminary MHD pressure drop analysis for the prototypical WCLL TBM with RELAP5/MOD3.3.
}

\author{
Melchiorri L., Narcisi V., Ciurluini C., Giannetti F., Caruso G., Tassone A. \\ DIAEE Nuclear Section - Sapienza University of Rome, Corso Vittorio Emanuele II, 244, 00186 Roma, Italy
}

\begin{abstract}
In fusion reactor development, the design of the breeding blanket (BB) represents an important technical challenge due to the extreme operative conditions that the component must withstand. One of the critical issues of a liquid metal BB is the occurring of magnetohydrodynamic (MHD) effects within the piping network that transports the electro-conductive fluid. Predicting MHD phenomena is fundamental for a reliable and efficient blanket design and computational tools are indispensable to achieve a fusion-relevant physical analysis. For this reason, a dedicated MHD module for the System Thermal-Hydraulic (STH) code RELAP5/MOD3.3 (RELAP5) is under development at Sapienza University of Rome. In its current state, RELAP5 can evaluate MHD pressure losses in many common layouts for BB piping system. In a previous work of the Authors, the verification and validation (V\&V) procedure of the MHD module has been discussed. In this paper, the code is used to assess the MHD pressure loss in the Water-Cooled Lithium Lead Test Blanket Module (TBM). Furthermore, the geometry of the TBM box is optimized to reduce the MHD pressure loss and to equalize the flow distribution among the Breeding Units.
\end{abstract}

Keywords: Magnetohydrodynamics (MHD), MHD pressure drop, systems code, RELAP5, breeding blanket, WCLL

\section{Introduction}

In the design of nuclear fusion reactors, liquid metals (LMs) are considered appealing working fluids due to their good features as coolant and tritium breeder/carrier. Considering their high electrical conductivity, whenever LMs flow in the presence of a strong magnetic field they are subjected to self-induced Lorenz forces that cause the onset of magnetohydrodynamic (MHD) effects. Those phenomena significantly alter the flow rate distribution, enhance pressure drops and deeply impact turbulent and heat transfer mechanisms, in turn affecting the blanket performances [1]-[3]. For those reasons it is important to develop numerical codes that can predict these effects so that it would be possible to guarantee a reliable and consistent design of the LM breeding blanket (BB).

In general, MHD blanket-scale analyses are carried out via semi-analytical approach, in which empirical and semi-empirical correlations are integrated with data extrapolated by direct numerical simulations [4]-[6]. However, this procedure results extremely time-consuming and not flexible in its scope but, on the other hand, a dedicated MHD code able to simulate all the phenomena involved in a liquid metal blanket is not yet available. As matter of fact, some computational fluid dynamic (CFD) software have dedicated MHD modules (e.g. OpenFOAM [7]), but unfortunately reactor-scale analysis is not currently achievable with CFD codes since it would be prohibitive, or utterly unfeasible, in terms of computational resources. Conversely, systems thermal-hydraulic (STH) codes could provide the efficient and quick simulation of the blanket at a component level but, currently, they have limited or non-existent MHD capability. Some examples of MHD codes implementing limited modelling features can be found in Ref. [8]-[12]. In this framework, a modified version of RELAP5/MOD3.3 (RELAP5) is under development at Sapienza University of Rome, which can handle MHD pressure drop in pipes and complex geometries [13]. Considering its ongoing features RELAP5 is already competitive, in terms of capabilities, with other STH codes that are able to model MHD phenomena. A recent review of MHD modelling in STH codes can be found in Ref. [14]. 
The scope of this work is to demonstrate that the RELAP5 module for MHD pressure drop, presented and validated in [13], is successfully able to carry out an actual design analysis. The study performed is focused on the simulation and optimization of the LM flow path in the Water-Cooled Lithium Lead (WCLL) Test Blanket Module (TBM), one of the test sections that are going to be used to gather data about blanket concepts during ITER experimental campaigns [15].

The outcome of detailed numerical analyses about the TBM manifold, described in Ref. [16], and breeding zone, discussed in Ref. [17], are used to assess the quality of the results obtained by our MHD subroutines. As in the Helium-Cooled Lithium Lead (HCLL) mock-up [18][19], which can be considered the predecessor of the WCLL design ${ }^{1}$, the manifold region is where the bulk of the pressure head loss in the module happens, whereas losses in the BZ are comparatively negligible. The prediction of pressure loss and flow distribution as calculated by RELAP5 for the TBM reference configuration is used as baseline to guide the optimization process. Two modifications of the manifold region reference design, first proposed in Ref. [16] to reduce the flow rate imbalance that is established among the breeding units (BUs), are then implemented in the RELAP5 model. Their effect on pressure loss and flow rate is assessed separately and jointly, alongside with further layouts proposed by the authors of this work.

\section{Theoretical background}

Whenever an electro-conductive fluid flows under the influence of a magnetic field, if its relative velocity is non null, electrical currents arise within the liquid domain. Those currents interact with the magnetic field themselves causing the appearance of Lorentz forces that oppose the fluid movement. To take in account the effect of those forces on the fluid velocity field, MHD governing equations are obtained by the combination of the Navier-Stokes' and Maxwell's equation sets. For LM MHD flows in fusion reactor circuits, it is possible to simplify the MHD governing equations using the induction-less or low magnetic Reynolds number assumption, which neglects the self-induced magnetic field and, therefore, reduces the complexity of the otherwise non-linear velocity/magnetic field coupling [1]. For the aim of this work, the attention is focused on the momentum balance equation in its non-dimensional form, where the MHD effect is represented by the addition of the Lorentz force as a source term on the right-hand side:

$$
\frac{1}{N}\left[\frac{\partial \mathbf{v}}{\partial t}+(\mathbf{v} \cdot \nabla) \mathbf{v}\right]=-\nabla p+\frac{1}{H a^{2}} \nabla^{2} \mathbf{v}+\mathbf{j} \times \mathbf{B}
$$

In equation (1), vectors $\mathbf{v}, \mathbf{j}$ and $\mathbf{B}$ stand for velocity, current density and magnetic field, respectively, and $\mathbf{p}$ is the pressure. Non-dimensional parameters are obtained by scaling with the mean velocity $v_{0}, j_{0}=\sigma v_{0} B_{0}$ and the external magnetic field magnitude $\mathrm{B}_{0}$; the non-dimensional pressure $p$ is scaled through $p_{0}=\sigma \mathrm{V}_{0} a \mathrm{~B}_{0}{ }^{2}$. The flow is governed by two fundamental parameters, namely the Hartmann number $\left(H a=a B_{0}(\sigma / \mu)^{0.5}\right)$ and the Stuart number, or interaction parameter $\left(N=\sigma a B_{0}^{2} / \rho v_{0}\right)$.

The square of $\mathrm{Ha}$ stands for the relative ratio of electromagnetic to viscous forces, whereas $N$ refers to the ratio of electromagnetic to inertial forces. The quantity $a$ is the typical length scale for the specific case of study, namely the half-length of a duct along magnetic field direction. Symbol $\sigma$ is the electrical conductivity of the fluid, whereas $\mu$ and $\rho$ denote dynamic viscosity and density of the fluid. Those parameters may be combined to return the Reynolds number in its classical formulation, so that $R e=H a^{2} / N=\rho v_{0} a / \mu$.

In general, $\mathrm{Ha}$ and $\mathrm{N}$ attain high values $\left(\approx 10^{4},[4]\right)$ in fusion blanket applications, so that viscous-less and inertia-less approximations are reasonable in most MHD flows. The flow is governed by the balance between

\footnotetext{
${ }^{1}$ Until 2017, the HCLL concept was considered for testing in the EU TBM program before being substituted by the WCLL due to a priority realignment in EUROfusion.
} 
Lorentz and pressure force in the central region of fluid domain, called core, whereas inertial and viscous effects are confined in thin fluid layers of thickness $\mathrm{O}\left(\mathrm{Ha}^{-1}\right)$ and $\mathrm{O}\left(\mathrm{Ha}^{-1 / 2}\right)$ close to solid walls perpendicular (Hartmann layers) or parallel (Side layers) to the magnetic field direction [1].

An additional fundamental non-dimensional parameter must be defined, i.e. the wall conductance ratio $c=$ $\sigma_{w} t_{w} / \sigma a$, to represent the tendency of the induced currents to close either through the fluid or the bounding walls. Symbols $\sigma_{w}$ and $t_{w}$ are the electrical conductivity of the duct walls and their thickness. It is worth mentioning that, in complex layouts as the one present in the WCLL TBM, different channels may have common walls; this feature causes the exchanging of electric currents across those walls. This phenomenon is referred as Madarame effect or electromagnetic coupling [20] and it can deeply impact the mass flow distribution, as well as the loss of load, in a liquid metal circuit. However, theoretical understanding of coupling is still limited, since the numerous variables involved in the phenomenon, and for this reason RELAP5 is currently not able to model the Madarame effect. Nevertheless, as extensively discussed in [13], the code can reasonably predict MHD pressure loss trends even for the complex systems typical of fusion applications. In general MHD pressure drops are treated with a hydrodynamic-like approach (see for example Ref. [4]), which considers the overall loss $\left(\Delta \mathrm{P}_{\mathrm{MHD}}\right)$ expressed as $\Delta \mathrm{P}_{\mathrm{MHD}}=\Delta \mathrm{P}_{2 \mathrm{D}}+\Delta \mathrm{P}_{3 \mathrm{D}}$. Two-dimensional $\mathrm{MHD}$ pressure drop $\left(\Delta \mathrm{P}_{2 \mathrm{D}}\right)$, or fully developed $\mathrm{MHD}$ flow drop, can be considered as the analogue of ordinary friction loss. This loss of load is due to Lorentz forces that are caused by electrical currents that close their path in a plane perpendicular to the flow direction. This kind of pressure drop has been studied in-depth, hence its characterization is supported by an exhaustive amount of data and extensively validated correlations are available in the literature [2][12][13].

Three-dimensional MHD pressure losses $\left(\Delta \mathrm{P}_{3 \mathrm{D}}\right)$, on the other hand, can be treated similarly to localized losses. They are caused by electrical current closing their paths in stream-wise direction, that arise whenever the flow runs into complex geometry elements (such as bends, cross-section variations, obstacles) and nonuniform electromagnetic boundary conditions, i.e. discontinuity in wall electrical conductivity and nonuniform magnetic field. All those are common situations in the liquid metal piping network of a fusion reactor. Unfortunately, data for 3D MHD pressure losses are relatively scarce compared with these available for fully developed MHD flow. As matter of fact, evaluating those kinds of losses is much more challenging since they strongly depend on the flow geometry and governing parameters. Anyhow, the most impactful features in the WCLL TBM layout, such as the influence on the MHD flow of expansion/contraction and bends, can be successfully modelled by RELAP5, as reported in [13].

\section{WCLL TBM geometry}

The WCLL TBM is going to be used in the experimental phase of ITER to gather data about a BB concept relying on a liquid phase low-pressure breeder being cooled by a high-pressure coolant, water in the present case [15][23].

A 3D view of the pre-conceptual TBM geometry is shown in Figure 1. The TBM is divided in two identical parallel sections; each section being composed of 8 vertically aligned breeding units (BUs), that are fed by a vertical conduit that represents the feeding manifold. The assembly of the $16 \mathrm{BUs}$ constitutes the BZ. After the fluid passes through the $\mathrm{BU}$, which flow path is sketched in Figure 2, it is collected in another vertical duct that is the draining manifold. The breeder is gradually conveyed from the feeding manifold to the BZ and, hence, its mass flow rate is reduced with the poloidal height, whereas the opposite happens in the draining manifold. The liquid metal undergoes a sequence of contractions and expansions in the manifold channels due to the presence of toroidally oriented stiffening plates that delimit each breeding unit and provide additional mechanical stability to the TBM box. In Figure 1, the inlet pipe and the outlet pipe of the TBM are not depicted; they are located, respectively, at the height of the bottom and top BU in a central position [24]. 


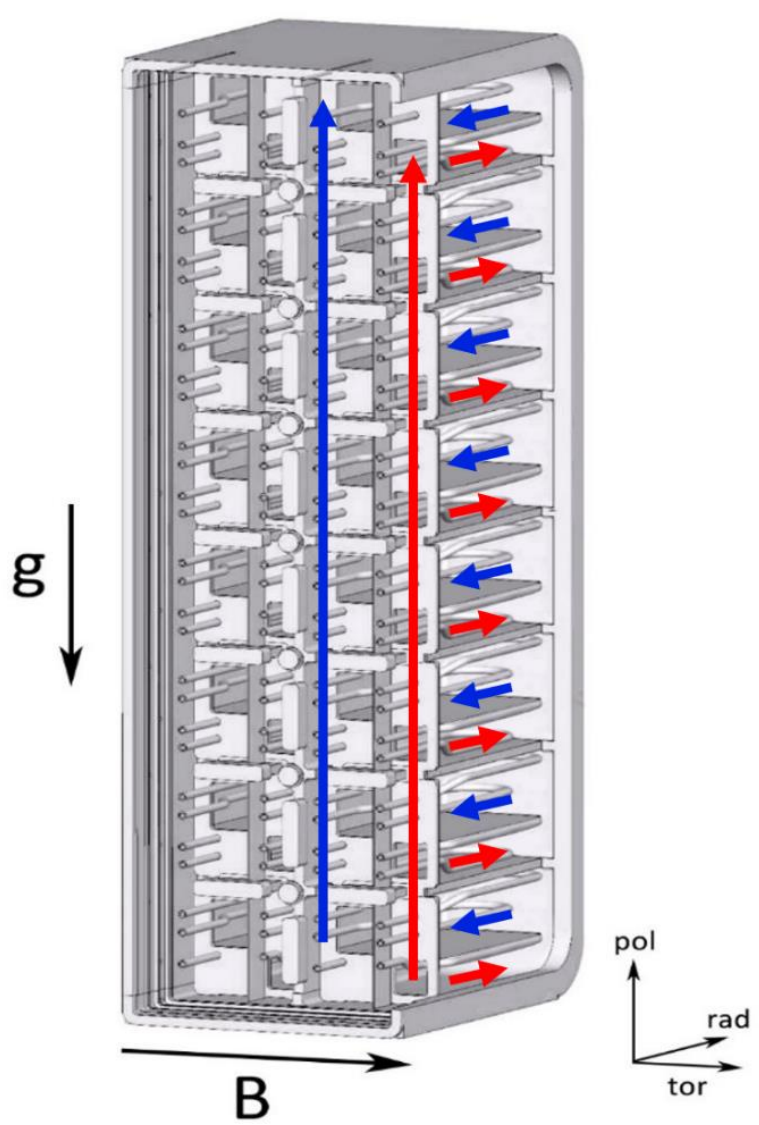

Figure 1. 3D model of the reference geometry, adapted from Ref. [16]. The Li-Pb hydraulic path is marked by arrows: red for the distribution leg, blue for the collection leg.

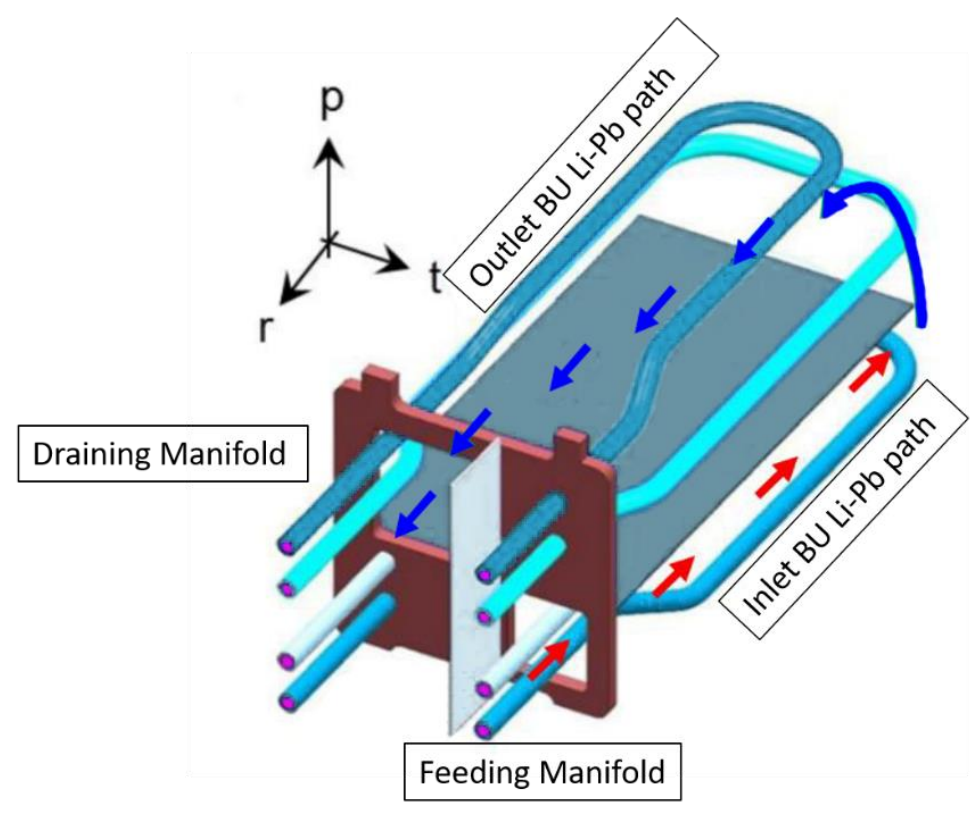

Figure 2. 3D CAD of a single Breeding Unit. Colored arrows outline the flow path [17].

In Figure 3, the red line points out the flow path in the inlet manifold, whereas the blue one is for the outlet manifold.

The Li-Pb alloy is the working fluid, and Eurofer97 is the structural material. Thermo-physical properties for these materials are evaluated at the reference temperature of $623 \mathrm{~K}$. Different values of magnetic field are studied $(0.5 \mathrm{~T}<\mathrm{B}<4 \mathrm{~T}$ ) which is imposed constant and unidirectional, oriented along $\mathrm{z}$ (toroidal) axis (see 
Figure 3). The poloidal field component, which is expected to account for approximately one-third of the toroidal one, is neglected. The BU toroidal half-width is chosen as characteristic length of the case, namely $a$ $=0.097 \mathrm{~m}$, while the reference velocity is taken as the mean value in a single $\mathrm{BU}$, in the range $0.1 \mathrm{~mm} / \mathrm{s}<v_{0}$ $<1 \mathrm{~mm} / \mathrm{s}$. The reference value selected for the RELAP5 model are $B=4 \mathrm{~T}$ and $v_{0}=0.1 \mathrm{~mm} / \mathrm{s}$, which are representative of the typical conditions foreseen for the experimental campaign to achieve the best relevancy with regard to the WCLL DEMO blanket [25]. Specifically, the chosen mean velocity leads to a total TBM mass flow rate of $0.12 \mathrm{~kg} / \mathrm{s}$. All dimensions of the model geometry are referred to the most recent WCLL TBM design [15]-[17] (Table 1).

In Figure 3 the alternative geometry configurations, proposed in [16], are summarized and are referred as Mod1 and Mod2. Mod1 consists in enlarging the gap between manifold sections, cutting the nonfunctional protuberance in the toroidal-radial stiffening plate (see Figure 3A). In Mod2, it is proposed to rearrange the toroidal position of the stiffening plate that separates feeding and collecting manifold, which is shifted stepwise in order to follow the change in mass flow rate that occurs along the poloidal direction (see Figure 3B). Moreover, modification of the layout that is suggested in this paper is depicted as Mod1b (Figure 3A). For Modb1, the orifices that ensure the hydraulic connection between manifold and BUs are tailored to the mass flow rate flowing in each $\mathrm{BU}$ in the reference configuration to achieve uniform flow distribution. As discussed in Section 5, authors proceeded to study the simultaneous effect of the aforementioned configurations, resulting in two additional layouts: Mod3 (Mod1 + Mod2) and Mod3b (Mod1 + Mod2 + Mod1b).

\begin{tabular}{|c|c|c|c|c|c|c|}
\hline Component & $\begin{array}{c}\text { Toroidal } \\
\text { width }[\mathrm{mm}]\end{array}$ & $\begin{array}{c}\text { Poloidal } \\
\text { height }[\mathrm{mm}]\end{array}$ & $\begin{array}{c}\text { Radial } \\
\text { length }[\mathrm{mm}]\end{array}$ & $\mathrm{t}_{\mathrm{w}}(\mathrm{Ha})[\mathrm{mm}]$ & $\begin{array}{c}\mathrm{t}_{\mathrm{w}} \text { (Side1) } \\
{[\mathrm{mm}]}\end{array}$ & $\begin{array}{c}\mathrm{t}_{\mathrm{w}}(\text { Side2) } \\
{[\mathrm{mm}]}\end{array}$ \\
\hline $\begin{array}{c}\text { Feeding } \\
\text { Manifold }\end{array}$ & 76 & 1610 & 60 & 30 & 15 & 15 \\
\hline $\begin{array}{c}\text { Draining } \\
\text { Manifold }\end{array}$ & 115 & 1610 & 60 & 30 & 15 & 15 \\
\hline $\begin{array}{c}\text { Breeding } \\
\text { Unit }(*)\end{array}$ & 193.5 & 190 & 430 & 30 & 30 & 30 \\
\hline
\end{tabular}

Table 1. Main geometrical parameters. $\left({ }^{*}\right)$ Stiffening plate not considered [15]-[17]. 


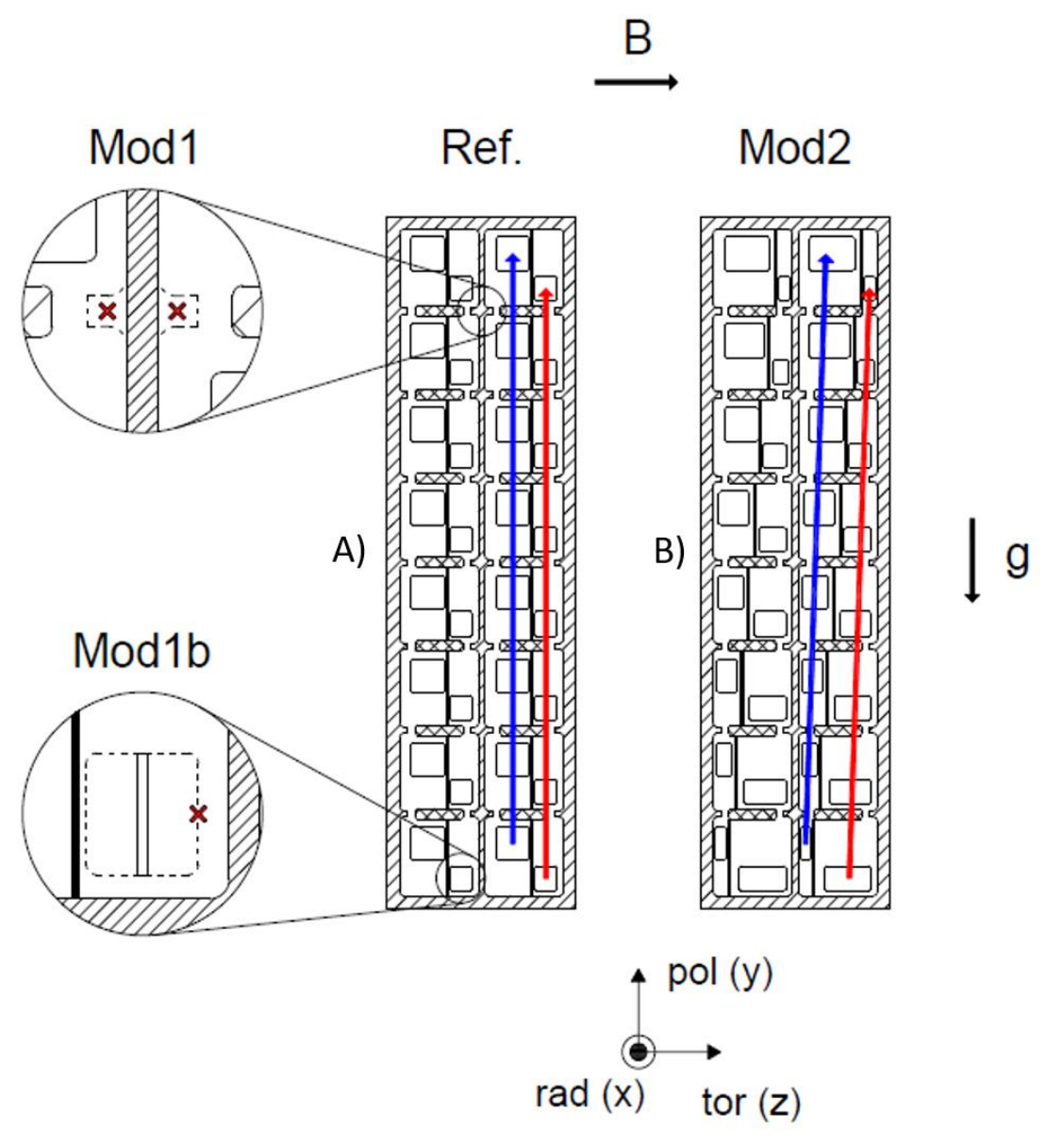

Figure 3. Poloidal-toroidal view of the TBM. Red and Blue arrows outline the LM flow path in feeding and collection manifold, respectively. A) the reference geometry is depicted, whereas are highlighted two modification proposed for the design optimization (Mod1 and Mod1b). B) another optimized configuration proposed in [16] (Mod2).

\section{Baseline configuration}

\subsection{Nodalization scheme}

Concerning the nodalization scheme employed (Figure 4), the WCLL TBM model has been derived from the model developed at Sapienza in the framework of the ITER WCLL Test Blanket System (TBS) Water Cooling System (WCS) analysis [26][27], which was already featuring Li-Pb volumes within the WCLL TBM, along with a section of the inlet and outlet ducts. The modelling presented in [27] has been improved following the guidelines obtained from the HCLL analysis [13]. Inlet and outlet thermodynamic conditions of the liquid metal (i.e., temperature and pressure) are fixed with 2 time-dependent volumes (TMDPVOL \#181 and \#220 in Figure 4). A time dependent junction (\#182) imposes the assumed mass flow rate through the circuit. Pipe component \#185 realizes the connection between the TMDPVOL and the two inlet manifolds. Each one is modelled with a pipe component composed of $23 \mathrm{CVs}$, reproducing the chambers which compose the manifold and their connections. Lateral junctions are used to couple the BUs with the corresponding volumes in the inlet and outlet plenum. Each BU is simulated with a pipe component consisting of $11 \mathrm{CVs}$. Outlet manifolds present the same modelling approach (slice modelling) used for the inlet ones, reflecting actual geometrical characteristics. The Li-Pb exiting the TBM is gathered within the outlet pipe (\#199) and drained in the TMDPVOL \#220. The two parallel sections of the WCLL TBM present the same geometrical features, except for the Hartmann wall thickness of the manifold channels. On the left flow path (blue arrow) in Figure 
$3 \mathrm{~A}$, the lateral plate of the TBM, which is thicker than the stiffening plates, is the Hartmann wall of the draining manifold, whereas the opposite happens for the feeding channel on the right flow path (red arrow).

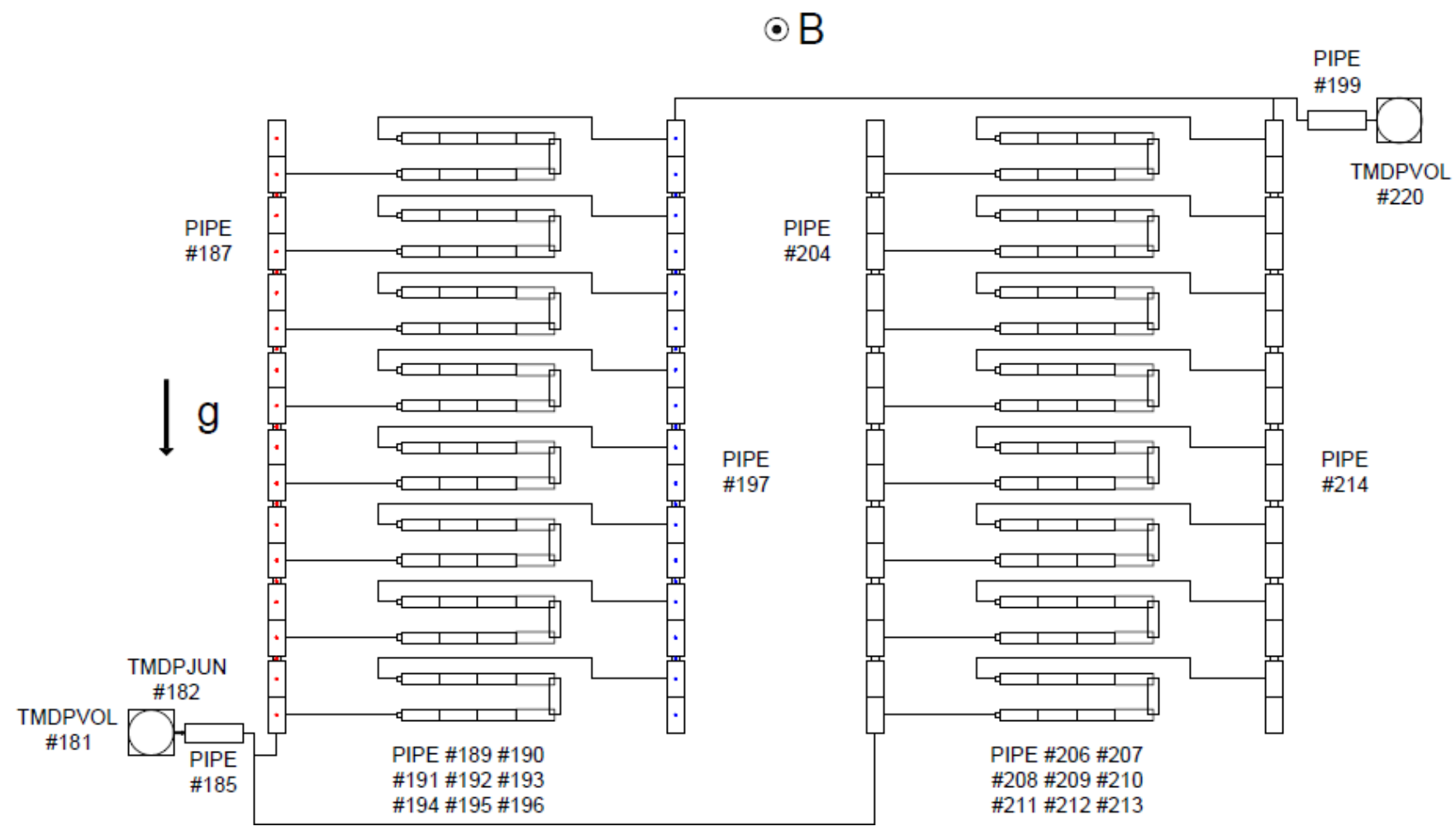

Figure 4. Nodalization scheme adopted for RELAP5 calculations. Red and blue dots underline the pressure data points for all the graphics shown in the following.

\subsection{Results and discussion}

Computational MHD analyses of the manifold highlighted how fluid velocity and pressure distribution, in such complicated geometry, are significantly affected by the occurrence of 3D MHD effects. Those phenomena are particularly intense where the flow meets path variation along magnetic field lines (toroidal variation). Namely, the gap between two portions of the manifold and the restriction/expansion at the manifold/BU interface gives a significant contribution to the pressure head loss in the TBM. Furthermore, the structural plate that separates feeding and draining manifold is electrically conducting; therefore, those fluid domains are electrically coupled. The two manifold conduits have different geometrical parameters, such as Hartmann wall thickness and cross-section. These conditions yield asymmetry in the velocity profiles, i.e. a large difference in the maximum value of velocity jet in opposite side layers. Additionally, since the pressure gradient is diverse in the two manifolds, where the mass flow rate is significantly different between feeding/draining parts, reversed flow regions appear. In other words, the velocity in the central portions of the manifolds has the same direction in both the ducts, while in the external parts, where the applied driving forces are differing, negative-velocity jets occur at the fluid layers parallel to B [16].

Focusing the attention on the pressure drop in the TBM is calculated that $\triangle \mathrm{p}_{\text {CFD }}=14 \mathrm{kPa}$ in reference conditions from CFD simulations [16]. The same calculation performed with RELAP5, adopting the nodalization scheme previously described, leads to $\Delta \mathrm{p}_{\mathrm{R} 5}=13.91 \mathrm{kPa}$. Albeit this outcome appears very promising, since the relative discrepancy is $\varepsilon<0.65 \%$, a further discussion is needed to highlight the uncertainty associated with our modelling approach. In reference [16], is clearly mentioned that $\Delta \mathrm{p}_{\text {CFD }}$ is exclusively referred to the pressure drop within the manifold, namely not considering the contribution of the breeding units' path, neither of the contraction/expansion at the manifold/BU interface that, conversely, are 
included in $\Delta \mathrm{p}_{\mathrm{R} 5}$. Furthermore, as explained in V\&V [13], RELAP5 cross-section variation model tends to overpredict MHD losses. On the other hand, the loss calculated in [16] is comprehensive of electro-coupling effects that, due to the co-flow configuration, are likely to increase the pressure drop due to the significant flow imbalance existing between the feeding and draining channels for the bulk of the manifold poloidal extension. As already discussed in [13], RELAP5 is not capable in its current state to recreate the effect of these phenomena on pressure loss. Therefore, the overall loss of load is similar between the two calculations because the lack of a coupling model in RELAP5 is compensated by the conservative behavior of 3D MHD model and BUs path inclusion in the analysis. However, it should be highlighted that the contribution of this last component is expected to be minimal $(0.3-0.5 \mathrm{kPa})$, as reported by detailed CFD analyses presented in Ref. [17].

Nevertheless, it is not a stretch to state that the code is able to predict with acceptable accuracy the trend of MHD pressure drop in this complex liquid metal circuit and, moreover, provides a reasonable figure for the pressure loss in the module.

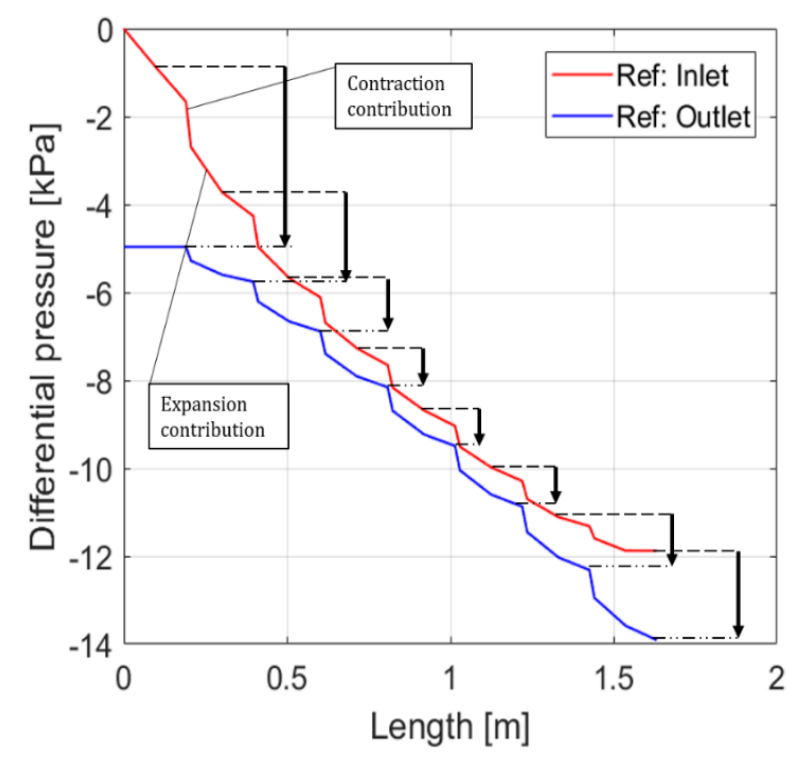

a)

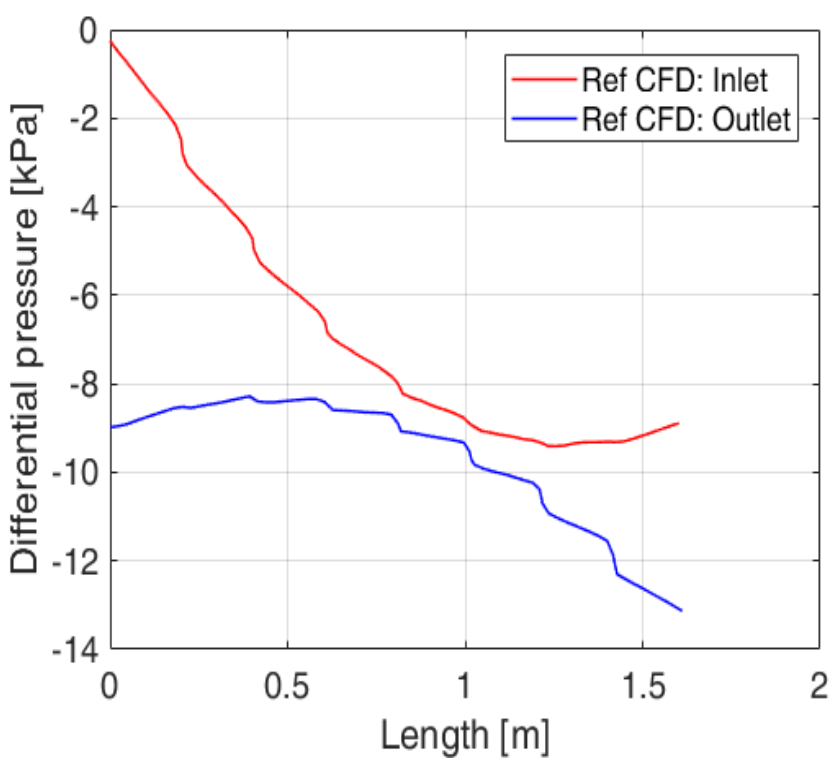

b)

Figure 5. a) Pressure profile in the manifold of Reference geometry versus its vertical height. In red is the feeding manifold and in blue the collecting one. Furthermore, the contribution of the contraction/expansion is outlined. Black arrows mark the pressure head available for each BU. b) Prediction of pressure profiles in the same geometry according to CFD calculation, data taken from Ref. [16].

In Figure 5, the pressure reduced by the hydrostatic head is plotted as a function of the vertical poloidal direction, i.e., the whole length of the manifolds. The distance between the red and blue lines (black arrows in Figure 5a) points to the pressure difference between feeding and draining manifold or, in other words, the available pressure head between entrance and exit of the corresponding BU. The additional threedimensional pressure drop arising at the connection between two portions of the manifolds is depicted by the steps visible in the pressure distribution, where the 2D MHD pressure gradient is increased due to the occurrence of sudden contractions and expansions occurring in the manifold. As can be observed, the pressure loss within the BUs differs for each unit (Figure 6). This is caused by the much higher flow rate occurring in the top (BU8) and bottom (BU1) channels, especially the latter, compared with the central one. It should be noted that this imbalance is uniquely a product of the manifold design, which results in a lower available pressure head for the central BUs that, consequently, receive less flow rate. Such a disequilibrium would be further magnified by electro-coupling phenomena, as can be observed by the comparison of Figure $5 a$ and Figure $5 b$, where in the latter we report some data taken from a recent CFD calculation. In the BU1 
and BU8, where the mass flow rate is higher, the loss of load is roughly two times the one that occurs in our RELAP5 model where the coupling is neglected. Nevertheless, RELAP5 is successful in recreating the qualitative trend of the pressure head along the poloidal coordinate so that one may reasonably expect that the computed flow rate distribution in Figure 6 will be close to one calculated by a more sophisticated CFD approach, even if affected by a certain error margin due to the coupling contribution. This fact has motivated us to attempt the use of RELAP5 to optimize the TBM geometry and reduce the flow imbalance.

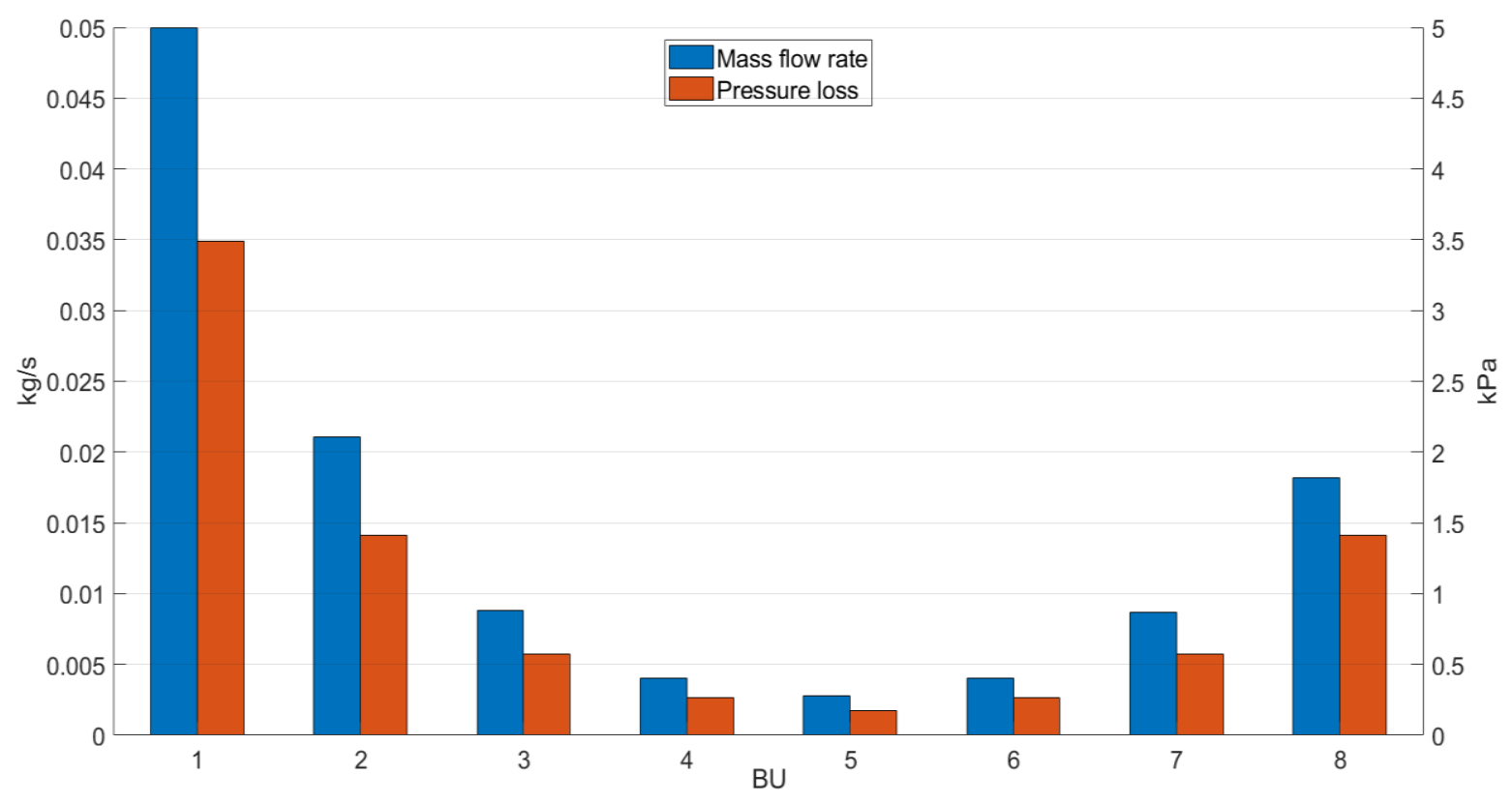

Figure 6. Bar plot comparing pressure loss and mass flow rate for each breeding unit for Reference configuration.

\section{Effect of WCLL TBM geometry on pressure loss and flow rate}

It is desirable to ensure that the same pressure head is available for each $\mathrm{BU}$ in order to achieve a uniform fluid distribution across them and avoid a harmful accumulation of tritium inventory due to stagnating breeder. Neglecting coupling effect between BUs, this would be possible only if the pressure along both manifolds varied linearly and the gradient was the same [16]. In the current manifold concept, the crosssections of the two conduits are different and remain constant along the entire poloidal length of the module. Consequently, the feeding manifold, which features a smaller cross-section and larger velocity, is generally characterized by a greater pressure loss than the larger draining manifold [16].

A more balanced mass flow rate among breeding units could be achieved if the TBM geometry was optimized, both for manifold and BU paths. Therefore, two variations to the module design are proposed in [16] to pursue this goal, referred as Mod1 and Mod2 (see Section 3). These two geometry modifications are studied separately in RELAP5 and their effect on the pressure loss is assessed. Consequently, as already mentioned, they are combined in a configuration labelled Mod3 to assess how the pressure loss is affected by the simultaneous presence of both geometry alterations.

In Table 2 are collected the main results in terms of total pressure drops and mass flow rate distribution for these alternative configurations are compared with the baseline TBM geometry discussed in Section 4.2. Mod1 has a positive effect on the total pressure losses with a $25 \%$ reduction, whereas in Mod 2 we observe a slight pressure drop increase (about $3 \%$ ). This is caused by the generally larger cross-section variation ratio (up to 50\%) that this modification introduces between manifold sections that, consequently, produces an increase in the 3D loss from cross-section variation. Conversely, the behavior of Mod3 is aligned with Mod1 since the modification in the stiffening plate geometry alleviates the increased loss from cross-section 
variation that was responsible for Mod2 behavior. In Figure 7 and Figure 8, it is shown how the pressure drop trend in the manifold is modified depending on the geometrical configuration.

Considering the flow distribution, it can be observed that, compared to the reference geometry case, the mass flow rate imbalance is just slightly alleviated. The best performances in this regard are observed for Mod3, but the flow imbalance is still significant. Even if it is effective in reducing the overall pressure loss, the alteration of the manifold geometry is not enough to achieve uniform flow distribution, at least if coupling is neglected.

For this reason, the additional geometry modification Mod1b is proposed, focusing on the manifold/BZ interface as described in Section 3. The approach which Mod1b concept relies on is the following: $a$ target flow distribution, $\Gamma_{t}=\Gamma / n \approx 12 \%$, is defined where $\Gamma$ is half the TBM flow rate, since the two flow paths are symmetric, and $n=8$ is the number of BUs in a flow path. If the mass flow rate in a BU is larger than the target, $\Gamma_{\mathrm{i}}>\Gamma_{t}$, the orifice is made smaller, whereas the opposite is done if $\Gamma_{\mathrm{i}}<\Gamma_{t}$. The process is repeated until uniform flow distribution is achieved. Moreover, the same methodology is applied to Mod3, in order to assess its effect on an already optimized configuration, which results in the layout Mod3b.

\begin{tabular}{|l|l|l|l|l|l|l|l|}
\hline Parameter & Unit & $\begin{array}{l}\text { Reference } \\
\text { design }\end{array}$ & Mod1 & Mod2 & Mod3 & Mod1b & Mod3b \\
\hline Total $\mathrm{Ap}$ & $\mathrm{kPa}$ & 13.91 & 10.42 & 14.35 & 10.41 & 20.7 & 13.23 \\
\hline Flow rate BU1 & $\%$ & 42.517 & 38.455 & 35.708 & 26.270 & 11.90476 & 11.905 \\
\hline Flow rate BU2 & $\%$ & 17.942 & 18.712 & 20.271 & 19.294 & 12.15986 & 11.990 \\
\hline Flow rate BU3 & $\%$ & 7.483 & 9.099 & 10.008 & 13.092 & 12.07483 & 12.160 \\
\hline Flow rate BU4 & $\%$ & 3.401 & 4.893 & 4.919 & 8.785 & 11.81973 & 11.990 \\
\hline Flow rate BU5 & $\%$ & 2.381 & 3.519 & 3.477 & 6.891 & 12.32993 & 12.245 \\
\hline Flow rate BU6 & $\%$ & 3.401 & 4.292 & 4.835 & 7.063 & 12.32993 & 12.245 \\
\hline Flow rate BU7 & $\%$ & 7.398 & 7.554 & 8.227 & 8.527 & 12.67007 & 12.160 \\
\hline Flow rate BU8 & $\%$ & 15.476 & 13.476 & 12.55 & 10.077 & 12.5 & 11.905 \\
\hline
\end{tabular}

Table 2. Percentage of the total mass flow rate crossing each breeding units (BU 1-8) for different configuration and total associated pressure drop.

Both Mod1b and Mod3b, as expected, provide a more satisfactory solution in terms of flow rate distribution. In Table 2, it is possible to observe that the two optimized layouts increase the overall pressure loss compared with Mod3, whereas the manifold pressure profile is shown in Figure 9 and Figure 10. Layout Mod1b features nearly double the pressure loss compared with Mod1 and it is a significant increase even compared with the reference configuration. Conversely, the optimized manifold layout in Mod3b, while still being penalized by a larger loss compared with Mod3, is characterized by a figure which is comparable with the reference configuration and achieves a nearly uniform flow distribution. As such, a geometry revision of the TBM based upon Mod3b is suggested to optimize the Li-Pb flow path. 


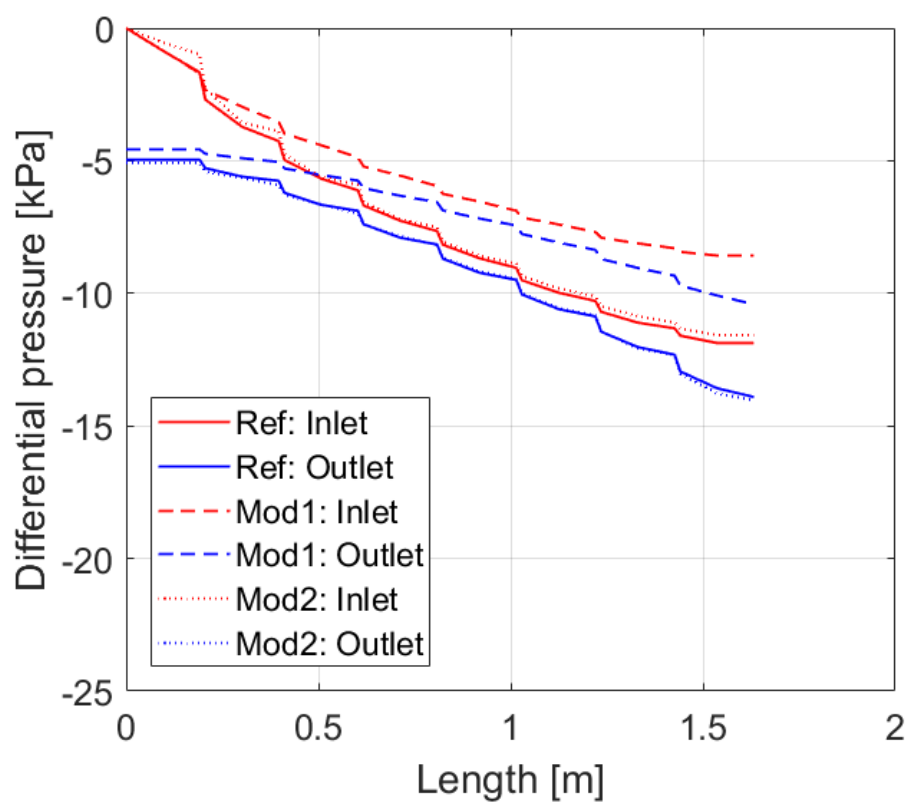

Figure 7. Pressure drop in feeding/draining manifold (red/blue) versus poloidal length of the module. The comparison is realized for three geometries: Reference geometry (full line), Mod1 (dashed line) and Mod2 (dotted line).

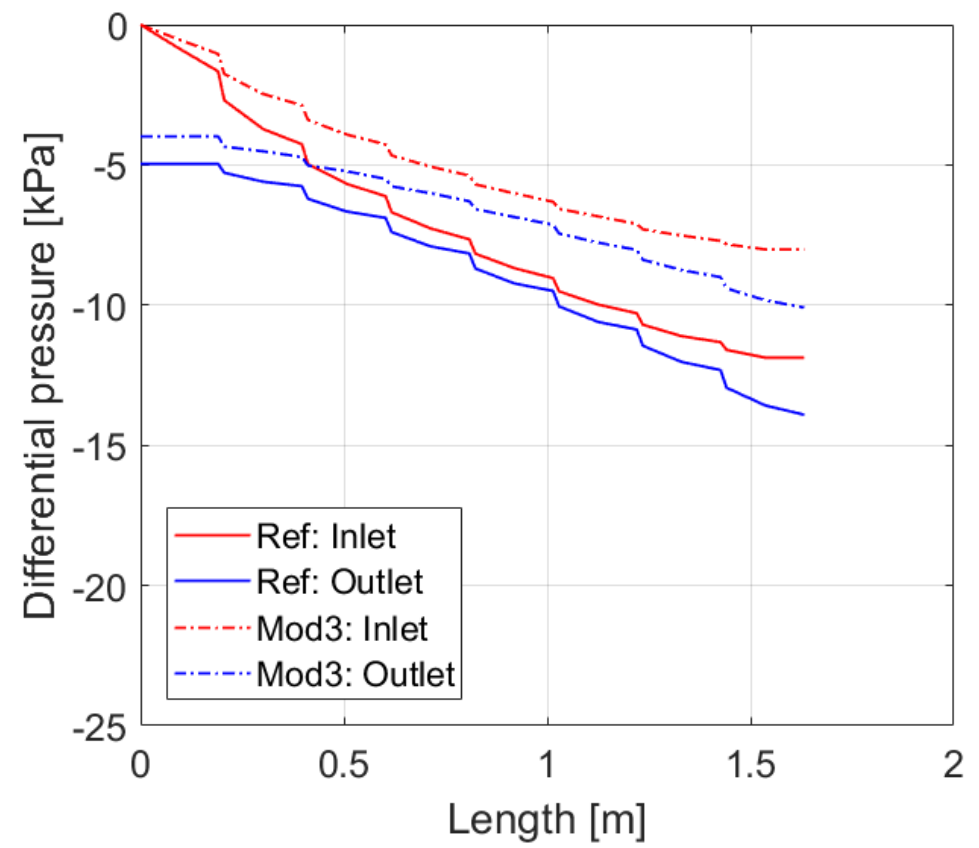

Figure 8. Pressure drop in feeding/draining manifold (red/blue) versus poloidal length of the module. The comparison is realized for two geometries: Reference geometry (full line), Mod3 (dashed line). 


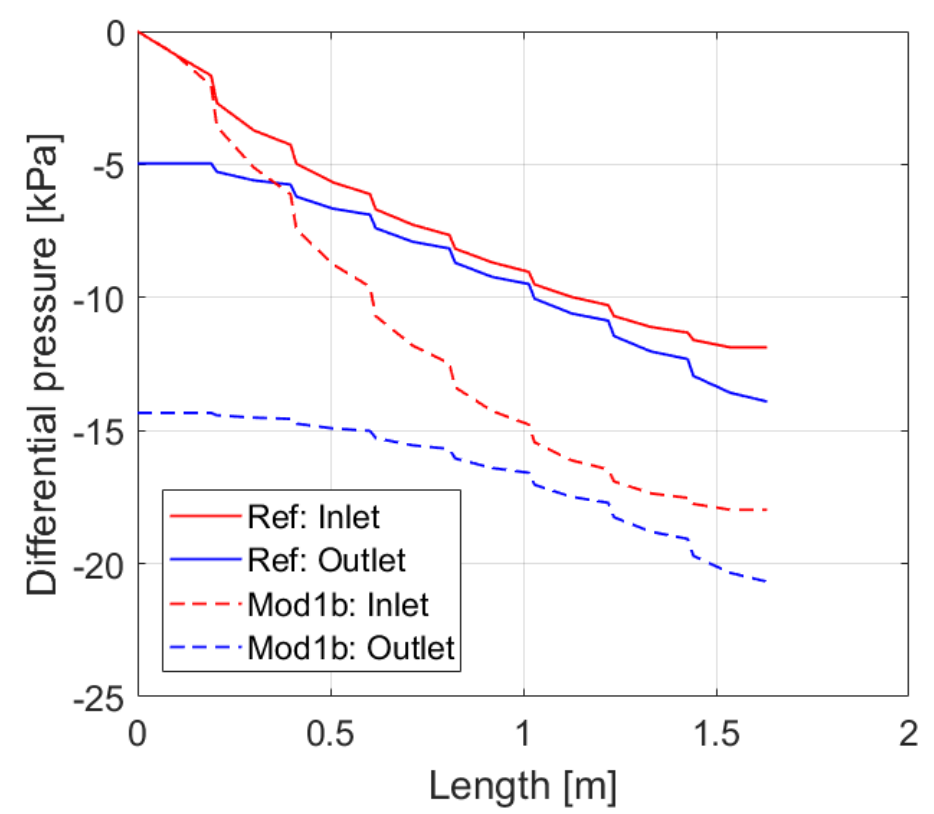

Figure 9. Pressure drop in feeding/draining manifold (red/blue) versus poloidal length of the module. The comparison is realized for two geometries: Reference geometry (full line), Mod1b (dashed line).

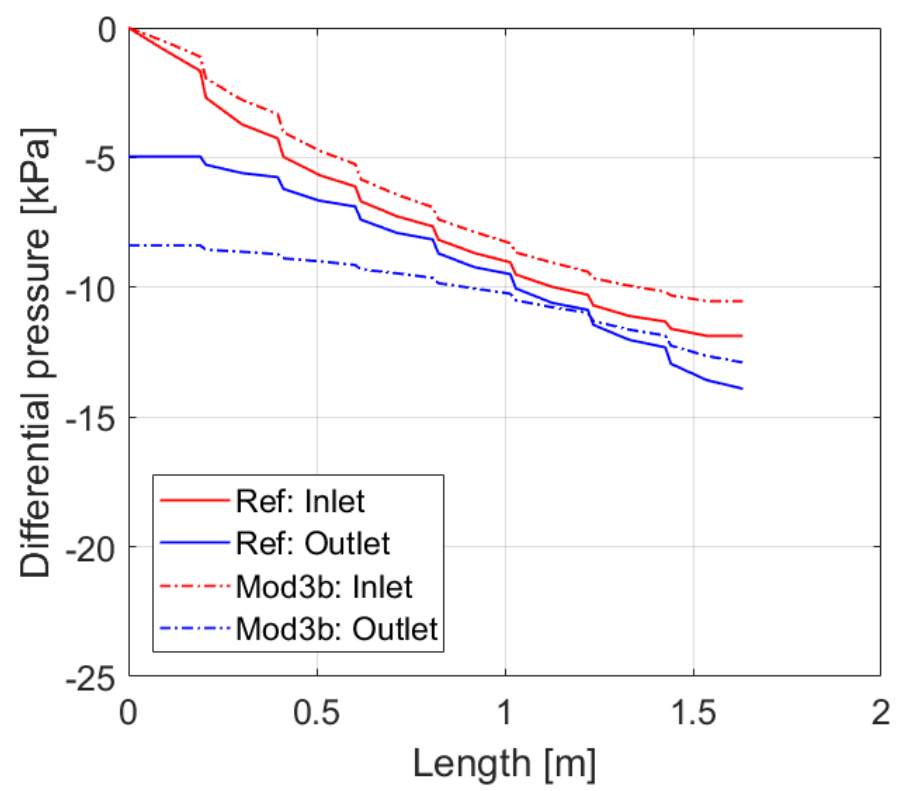

Figure 10. Pressure drop in feeding/draining manifold (red/blue) versus poloidal length of the module. The comparison is realized for two geometries: Reference geometry (full line), Mod3b (dashed line).

As a final note, it should be highlighted that the flow distribution in the TBM is always going to be affected by the electromagnetic coupling between the BUs and, as such, even a geometry based on Mod3b is unlikely to achieve perfectly uniform flow distribution but, rather, like in the experiments carried on the HCLL mockup, it will feature an excess flow rate in the terminal channels, i.e. BU1 and BU8 [19] [28]. Nevertheless, a pre-existing flow imbalance due to differing available pressure heads across the BUs is only going to magnify the coupling effect, which is known to be affected by disparity in flow rate, that is to say mean velocity, in adjacent channels, as it was underlined in [13], where a validation exercise with this feature. is discussed. The optimized geometry proposed in this work is going to avoid any flow imbalance due to baseline MHD 
losses and, at the bare minimum, will make possible to minimize coupling effect both in terms of additional pressure loss and induced non-uniformity in the flow distribution.

\section{Conclusions and further works}

New subroutines for the STH code RELAP5/MOD3.3 have been developed at Sapienza University of Rome to estimate the MHD pressure loss in liquid metal with the final goal to create a numerical tool that could predict all MHD effects that are characteristic of LM breeding blankets. This code is here used for the assessment of the MHD pressure loss in the WCLL TBM and the optimization of the Li-Pb hydraulic path therein.

Referring to the detailed numerical analyses presented in [16], it is first demonstrated that RELAP5 can quantitatively predict the total pressure drop within the module and qualitatively recreate the flow rate distribution between the BUs. Consequently, alternative geometrical configurations are investigated to assess their effect on flow distribution and pressure loss in the TBM box. The geometry that is proven to be the better option, in terms of mass flow rate balance and reduced pressure drops, is the so-called Mod3b. In this layout calibrated restrictions at the manifold/BU interfaces were added, with a toroidal stepwise shifting of the structural plate that divides feeding and collecting manifold to follow the decreasing and increasing flow rate in these hydraulic elements, and optimization of the openings between manifold sections.

It is crucial to underline that this assessment must be considered as a preliminary guideline to the TBM design, acknowledging that the code is still lacking some important capabilities as a best estimate tool, like electromagnetic coupling, which is currently under development [13]. However, this new version of RELAP5 can be regarded as a step in the right direction for the development of a fusion system code able to predict MHD phenomena.

In conclusion, RELAP5 has proven to be an efficient tool for magnetohydrodynamic pressure drop evaluations in liquid metal circuits. Further work has still to be done to reach an ideal implementation. Forthcoming activities will be focused on refining pressure drops correlations currently implemented, fine tuning a basic electro-coupling model already developed and, at last, deriving a suitable model to assess heat transfer phenomena in MHD environment. Concerning the WCLL TBM, it should be noted that our analysis neglected the effect of the poloidal field component, which is a conventional modelling choice, even if it can potentially affect both the 2D and 3D MHD loss estimate. A more refined analysis should include this aspect, as well as coupling.

\section{Acknowledgements}

The authors wish to thank Dr. Joelle Vallory and Dr. Italo Ricapito, for their preliminary review of the paper and effective suggestions. A heartfelt thanks to Ms. Elisa Martino, for her precious help with several images reported in this paper. This work has been carried out within the framework of the EUROfusion Consortium and has received funding from the Euratom research and training program 2014-2018 and 2019-2020 under grant agreement No 633053. The views and opinions expressed herein do not necessarily reflect those of the European Commission.

\section{REFERENCES}

[1] U. Muller and L. Bühler, Magnetofluiddynamics in Channels and Containers. Springer Science and Business Media, 2001.

[2] I. R. Kirillov, C. B. Reed, L. Barleon, and K. Miyazaki, "Present understanding of MHD and heat 
transfer phenomena for liquid metal blankets," Fusion Eng. Des., vol. 27, no. C, pp. 553-569, 1995, doi: 10.1016/0920-3796(95)90171-X.

[3] S. Smolentsev, R. Moreau, L. Bühler, and C. Mistrangelo, "MHD thermofluid issues of liquid-metal blankets: Phenomena and advances," Fusion Eng. Des., vol. 85, no. 7-9, pp. 1196-1205, 2010, doi: 10.1016/j.fusengdes.2010.02.038.

[4] A. Tassone, G. Caruso, and A. Del Nevo, "Influence of PbLi hydraulic path and integration layout on MHD pressure losses," Fusion Eng. Des., vol. 155, no. January, p. 111517, 2020, doi: 10.1016/j.fusengdes.2020.111517.

[5] A. Tassone, S. Siriano, G. Caruso, M. Utili, and A. Del Nevo, "MHD pressure drop estimate for the WCLL in-magnet PbLi loop," Fusion Eng. Des., vol. 160, no. June, p. 111830, 2020, doi: 10.1016/j.fusengdes.2020.111830.

[6] J. Reimann, G. Benamati, and R. Moreau, "Report of working group MHD for the blanket concept selection exercise (BSE)." Technical Report FZKA 5652, Kalrsruhe, 1995.

[7] S. Smolentsev et al., "An approach to verification and validation of MHD codes for fusion applications," Fusion Eng. Des., vol. 100, pp. 65-72, 2015, doi: 10.1016/j.fusengdes.2014.04.049.

[8] M. J. Wolfendale and M. J. Bluck, "A coupled systems code-CFD MHD solver for fusion blanket design," Fusion Eng. Des., vol. 98-99, pp. 1902-1906, 2015, doi: 10.1016/j.fusengdes.2015.04.025.

[9] A. Batti, "System-level hydraulic modeling of the PbLi loop for the Breeding Blanket of a tokamak." Master Thesis, Politecnico di Torino, 2020.

[10] D. Panayotov et al., "A Methodology for Accident Analysis of Fusion Breeder Blankets and Its Application to Helium-Cooled Lead-Lithium Blanket," IEEE Trans. Plasma Sci., vol. 44, no. 10, pp. 2511-2522, 2016, doi: 10.1109/TPS.2016.2607784.

[11] A. Grief et al., "Qualification of MELCOR and RELAP5 models for EU HCLL TBS accident analyses," Fusion Eng. Des., vol. 124, pp. 1165-1170, 2017, doi: 10.1016/j.fusengdes.2017.03.048.

[12] D. Panayotov et al., "Uncertainties identification and initial evaluation in the accident analyses of fusion breeder blankets," Fusion Eng. Des., vol. 136, no. September 2017, pp. 993-999, 2018, doi: 10.1016/j.fusengdes.2018.04.053.

[13] L. Melchiorri, V. Narcisi, F. Giannetti, G. Caruso, and A. Tassone, "Development of a RELAP5/MOD3.3 Module for MHD Pressure Drop Analysis in Liquid Metals Loops: Verification and Validation," Energies, vol. 14, 2021, doi: https://doi.org/ 10.3390/en14175538.

[14] C. Mistrangelo et al., "MHD R\&D Activities for Liquid Metal Blankets," Energies, vol. 14, 2021, doi: https://doi.org/10.3390/en14206640.

[15] J. Aubert et al., "Design and preliminary analyses of the new Water Cooled Lithium Lead TBM for ITER," Fusion Eng. Des., vol. 160, no. July, p. 111921, 2020, doi: 10.1016/j.fusengdes.2020.111921.

[16] C. Mistrangelo, L. Bühler, C. Koehly, and I. Ricapito, "Magnetohydrodynamic velocity and pressure drop in manifolds of a WCLL TBM," Nucl. Fusion, vol. 61, no. 9, 2021, doi: 10.1088/17414326/ac18dc.

[17] A. Tassone and G. Caruso, "Computational MHD analyses in support of the design of the WCLL TBM breeding zone," Fusion Eng. Des., vol. 170, no. 112535, 2021, doi: https://doi.org/10.1016/j.fusengdes.2021.112535.

[18] L. Bühler, H.-J. Brinkmann, S. Horanyi, and K. Starke, "Magnetohydrodynamic flow in a mock-up of a 
HCLL blanket. Part II. Experiments," no. FZKA 7424 Tech. Rep. EUROFusion. 2008.

[19] C. Mistrangelo and L. Bühler, "Electric flow coupling in the HCLL blanket concept," Fusion Eng. Des., vol. 83, no. 7-9, pp. 1232-1237, 2008, doi: 10.1016/j.fusengdes.2008.07.004.

[20] H. Madarame, K. Taghavi, and M. S. Tillack, "Influence of Leakage Currents on Mhd Pressure Drop.," Fusion Technol., vol. 8, no. 1 pt 2(A), pp. 264-269, 1985, doi: 10.13182/fst85-a40055.

[21] K. Miyazaki, S. Kotake, N. Yamaoka, S. Inoue, and Y. Fujii-e, "MHD Pressure drop of NaK flow in stainless steel pipe," Nucl. Technol., vol. 4, no. 2 pt 2, pp. 447-452, 1983, doi: 10.13182/fst4-2p2447.

[22] K. Miyazaki, S. Inoue, N. Yamaoka, T. Horiba, and K. Yokomizo, "Magneto-Hydro-Dynamic Pressure Drop of Lithium Flow in Rectangular Ducts.," Fusion Technol., vol. 10, no. 3 pt 2A, pp. 830-836, 1986, doi: 10.13182/fst10-830.

[23] A. Tincani et al., "Conceptual design of the main Ancillary Systems of the ITER Water Cooled Lithium Lead Test Blanket System," Fusion Eng. Des., vol. 167, no. January, 2021, doi: 10.1016/j.fusengdes.2021.112345.

[24] R. Boullon, J. Aubert, and A. Morin, "Definition of a WCLL reference TBM set design based on the WCLL BB design - First CAD model and scoping calculations," 2019, [Online]. Available: https://idm.euro-fusion.org/?uid=2N7CDT.

[25] A. Del Nevo et al., "Recent progress in developing a feasible and integrated conceptual design of the WCLL BB in EUROfusion project," Fusion Eng. Des., vol. 146, no. March, pp. 1805-1809, 2019, doi: 10.1016/j.fusengdes.2019.03.040.

[26] C. Ciurluini, V. Narcisi, A. Tincani, C. Ortiz Ferrer, and F. Giannetti, "Conceptual design overview of the ITER WCLL Water Cooling System and supporting thermal-hydraulic analysis," Fusion Eng. Des. vol. 171, no. 112598, 2021, doi: https://doi.org/10.1016/j.fusengdes.2021.112598.

[27] C. Ciurluini et al., "Thermal-hydraulic modeling and analysis of the Water Cooling System for the ITER Test Blanket Module," Fusion Eng. Des., vol. 158, no. April, p. 111709, 2020, doi: 10.1016/j.fusengdes.2020.111709.

[28] C. Mistrangelo and L. Bühler, "Determination of multichannel MHD velocity profiles from wallpotential measurements and numerical simulations," Fusion Eng. Des., vol. 130, no. November 2017, pp. 137-141, 2018, doi: 10.1016/j.fusengdes.2018.03.041. 